SNF-5953

Revision 1

\title{
PROCESS HOOD STAND SUPPORT STEEL
}

Prepared for the U.S. Department of Energy

Assistant Secretary for Environmental Management

Project Hanford Management Contractor for the

U.S. Department of Energy under Contract DE-AC06-96RL13200

Fluor Hanford

P.O. Box 1000

Richland, Washington 


\section{PROCESS HOOD STAND SUPPORT STEEL}

Project No: W-441

Document Type: RPT

Division: SNF

C Van Katwijk

$\mathrm{FH}$

Date Published

June 2000

Prepared for the U.S. Department of Energy

Assistant Secretary for Environmental Management

Project Hanford Management Contractor for the

U.S. Department of Energy under Contract DE-AC06-96RL13200

Fluor Hanford

P.O. Box 1000

Richland, Washington
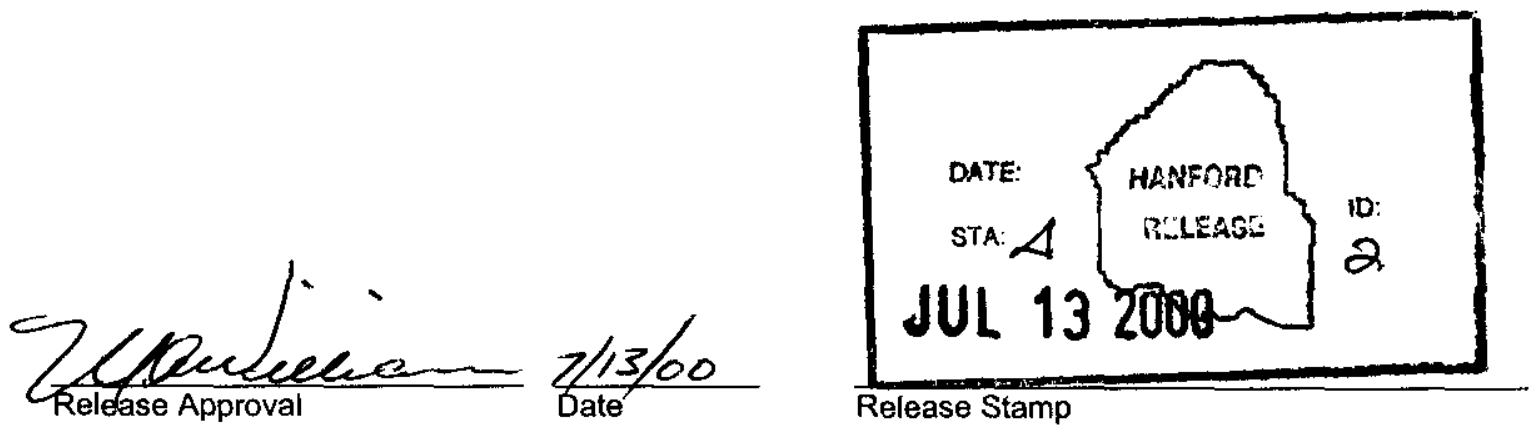
TRADEMARK DISCLAIMER

Reference herein to any specific commercial product, process,

or service by trade name, trademark, manufacturer, or

otherwise, does not necessarily constitute or imply its

endorsement, recommendation, or favoring by the United

States Government or any agency thereof or its contractors or subcontractors.

This report has been reproduced from the best available copy.

Printed in the United States of America

Total Pages: 14

SUF-5953, nWI 
RECORD OF REVISION

\section{(2) Title}

PROCESS HOOD STAND SUPPORT STEEL

Change Control Record

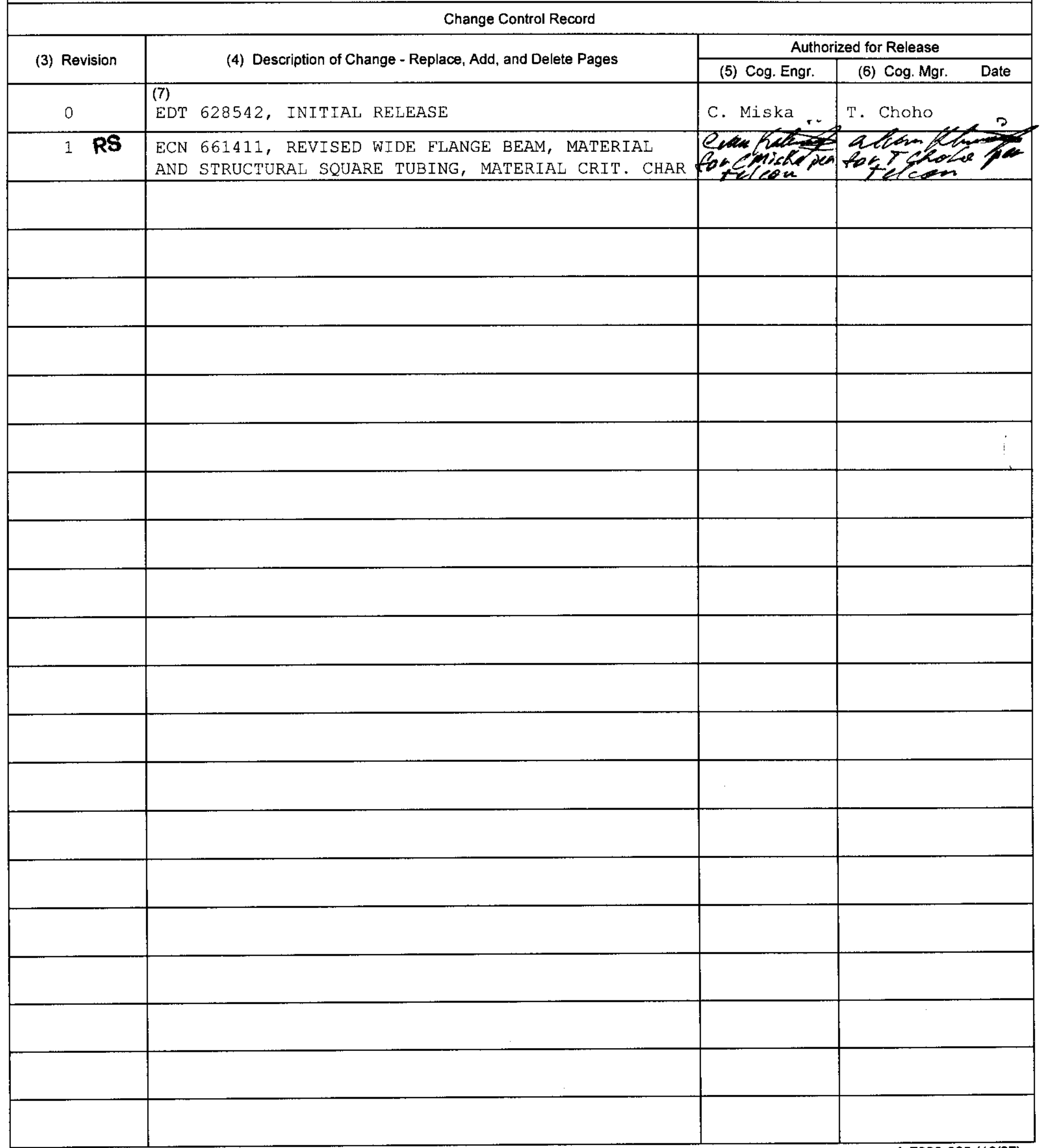


Commercial Grade Item Upgrade Dedication Form

ECN No. N/A CGINo. CGI-SNF.D-3-C1-057

Title: Process Hood Stand Support Steel

SNF-5953, Rev. 1

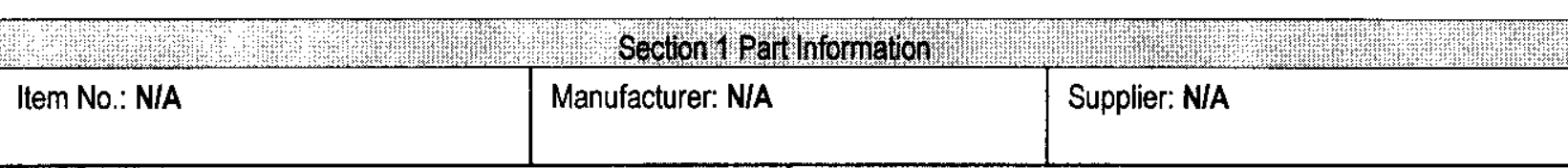

Mfg. Part/Model No.: NIA

Supplier's P/N: N/A

Part Description: N/A

End Use Description: N/A

\begin{tabular}{|c|c|c|c|}
\hline W" & Section $2 \mathrm{a}$ & hent linformation & Whing \\
\hline $\begin{array}{l}\text { Equipment No: } \\
\text { See attached Section } 9\end{array}$ & $\begin{array}{l}\text { Specification No:: } \\
\text { SNF-6209, Rev. } 0 \\
\text { (W-441-C1) }\end{array}$ & $\begin{array}{l}\text { Manufacturer: } \\
\text { Various, See Section } 9\end{array}$ & $\begin{array}{l}\text { Past P.O. No.: } \\
\text { N/A }\end{array}$ \\
\hline $\begin{array}{l}\text { Procurement and/or } \\
\text { Model No.: See attached Section } \\
9\end{array}$ & \multicolumn{2}{|c|}{$\begin{array}{l}\text { Equipment Supplier (if different from manufacturer): } \\
\text { Monarch Machine and Tool }\end{array}$} & $\begin{array}{l}\text { Equip. Supplier's Part No.: } \\
\text { N/A }\end{array}$ \\
\hline
\end{tabular}
tubing.

Section 26 commercial Availability of the ltem

1. Is the Item available from a catalogue from a qualified NQA1 supplier or ISO 9000 supplier (coordinate with project CGI interface Engineer or BTR)? [ ] YES (go to \#2 below) [X] NO (go to procedure step 6.3.2, proceed to dedicate ltem) If not available from a qualified NQA1 supplier, is it available from an ISO 9000 supplier? (coordinate w/ project CGI Interface Engineer or BTR):

[ ] YES (go to \#2 below, procedure step 6.3.2, dedicate Item) [X] NO (procedure step 6.3.2, dedicate Item)

2. List of Candidate qualified suppliers or ISO 9000 suppliers: N/A

3. Recommended Procurement Strategy(coordinate with project CGl interface Engineer or BTR): N/A

\section{Section 2 C CG1 Determination}

CGI Determination Questions:

$\# 1$ : Is the ltem subject to design or specification requirements that are unique to nuclear facilities or activities?

[ ] YES (the Item is not commercial grade) [X] NO (continue)

\#2: Is the Item used in applications other than nuclear facilities or activities?

[ ] NO (the item is not commercial grade) [X] YES (continue)

$\# 3$ : is the Item ordered from manufacturer/supplier on the basis of specifications set forth in the manufacturer's catalog?

[ ] NO (the item is not commercial grade) [X] YES (continue)

[X] All three criteria have been satisfied. The Item meets the definition of commercial grade.

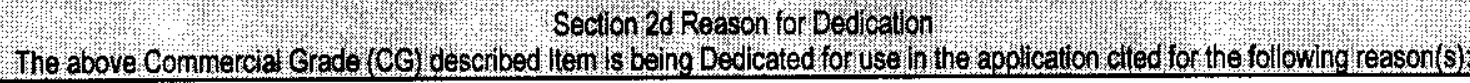

\begin{tabular}{c|l}
$X$ & Item is being purchased from a non-ESL manufacturer supplier as CG to be used in a Safety Class application. \\
\hline
\end{tabular} Item is being purchased from a non-ESL manufacturer supplier as CG to be used in a Safety Significant application.

Item was purchased from a non-ESL manufacturer supplier as CG to be used in a Safety Class application.

Item was purchased from a non-ESL manufacturer supplier as CG to be used in a Safety Significant application.

Other ('like-for-like', similar, substitution, replacement evaluation) 


\section{Section 3 Failure Effects Evaluation}

A. Part/Component Safety Function:

1. Provide supporting structure for the vacuum drying process hood.

2. Maintain critical function before, during, and after Seismic Event.

B. Part/Component Functional Mode:

Safety Function \#1: [ ] Active [X] Passive

Safety Function \#2:[ ] Active [X] Passive

Safety Function \#3: [ ] Active [ ] Passive

Active - Mechanical or Electrical change of state is required to occur for the component to perform its safety function

Passive - Change of state is not required for the component to perform its safety function

C. Host Component Safety Function (if applicable): N/A

1.

D. Failure Mode(s) and the effects on component or system safety function (see Worksheet 1):

1. Fracture, material fatigue, bolting/weld failure, could result in inadequate support for the process hood.

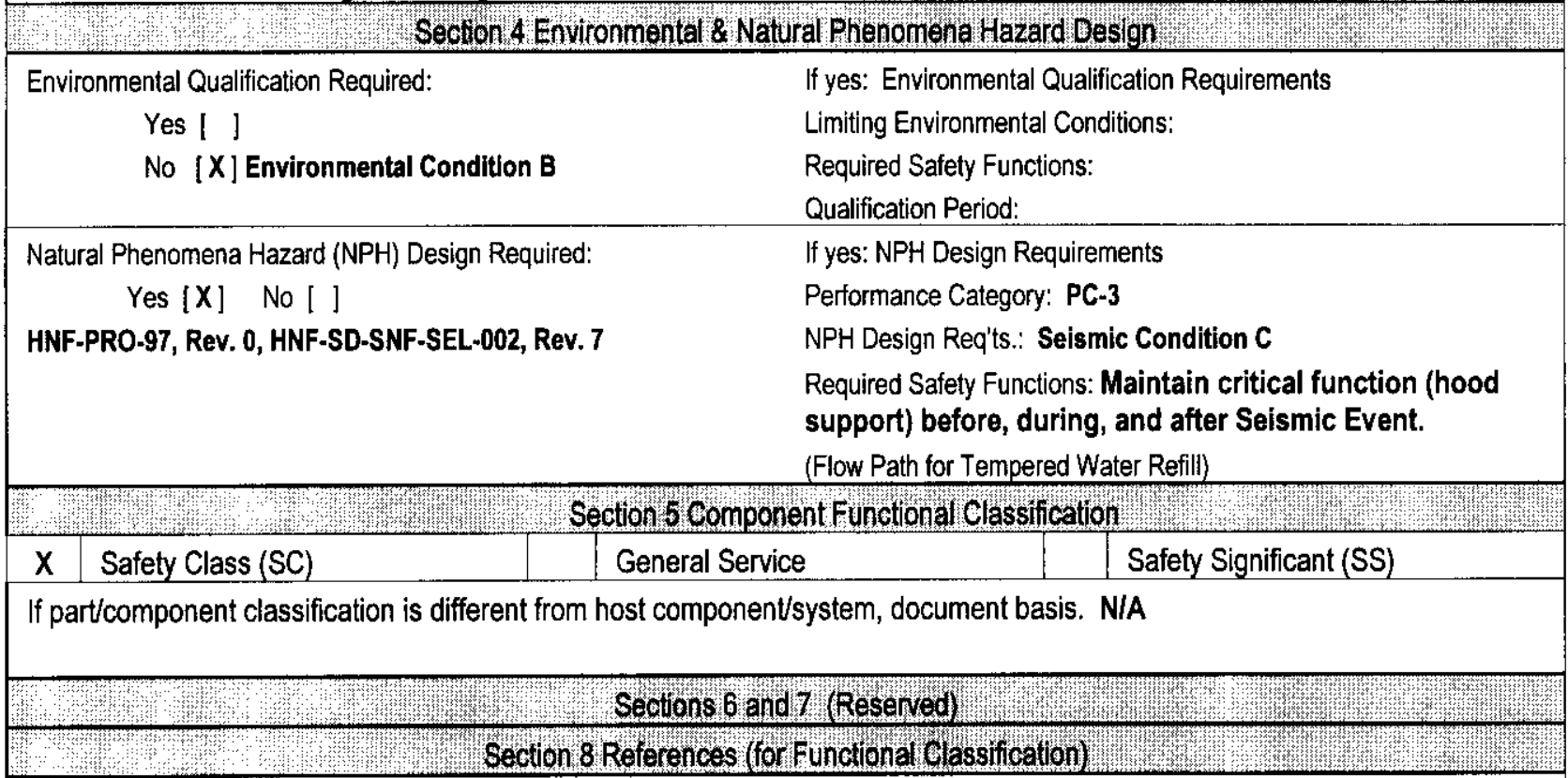

National Codes/Standards: ASTM A 500-93, Purchase order to request manufacturer's certification that the material was manufactured and tested in accordance with this specification. A report of the chemical and tensile test shall be furnished.

Safety Analysis Report (SAR): HNF- 3553, Rev. 0a, Annex B

Drawings: H-1-83980, Sheets 1-3, HNF-SD-SNF-SEL-002, Rev. 7

Vendor Manual/Manufacturer/Supplier Information: Catalog Cut Sheets: Ryerson Stocks and Services, Square Structural Tubing, Carbon Steel; I-Beams; I-Beams - Wide Flange Beams 


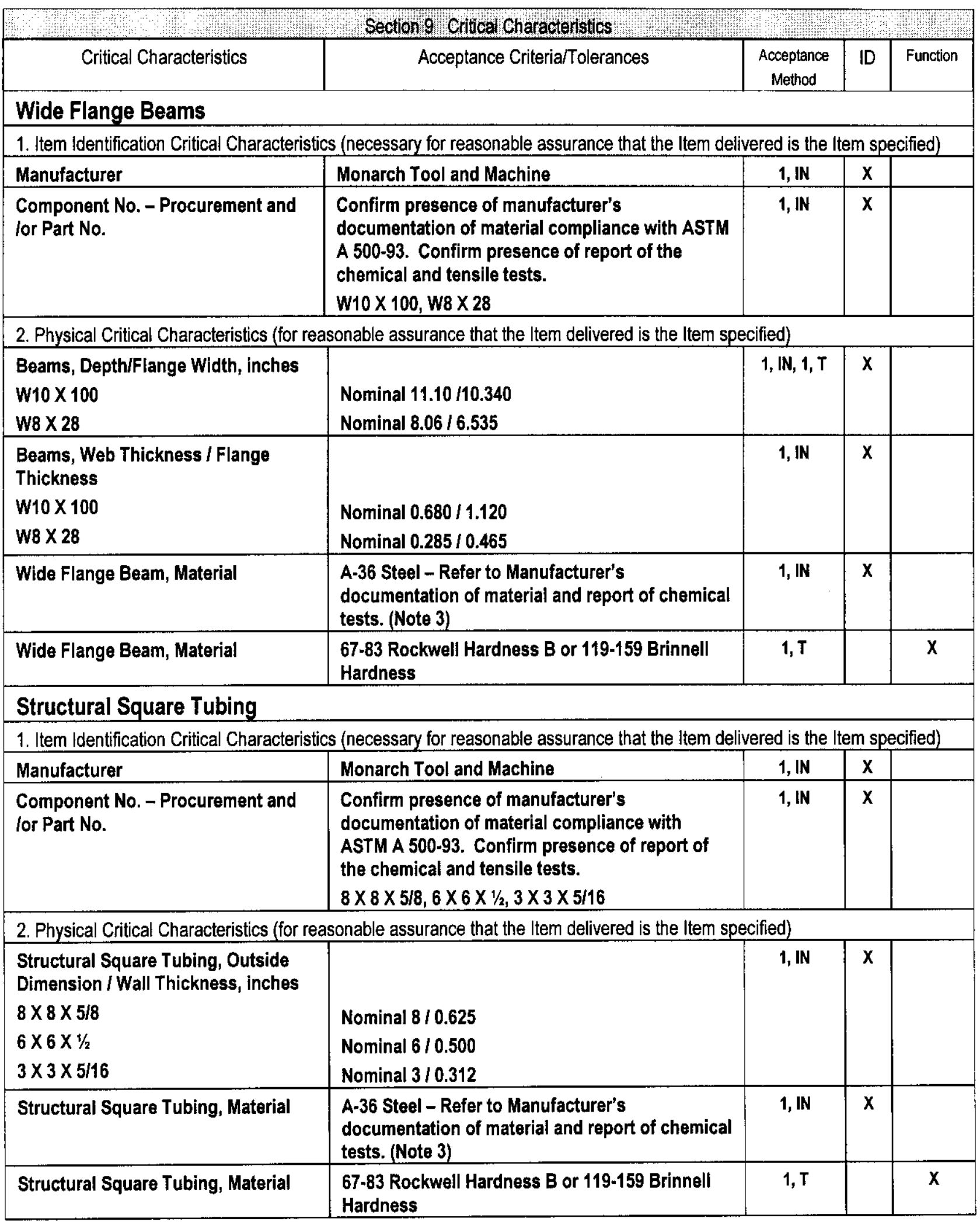


Commercial Grade Item Upgrade Dedication Form

ECN No. N/A CGI No. CGI-SNF-D-3-C1-057

Page 4 of 10

Tittle: Process Hood Stand Support Steel

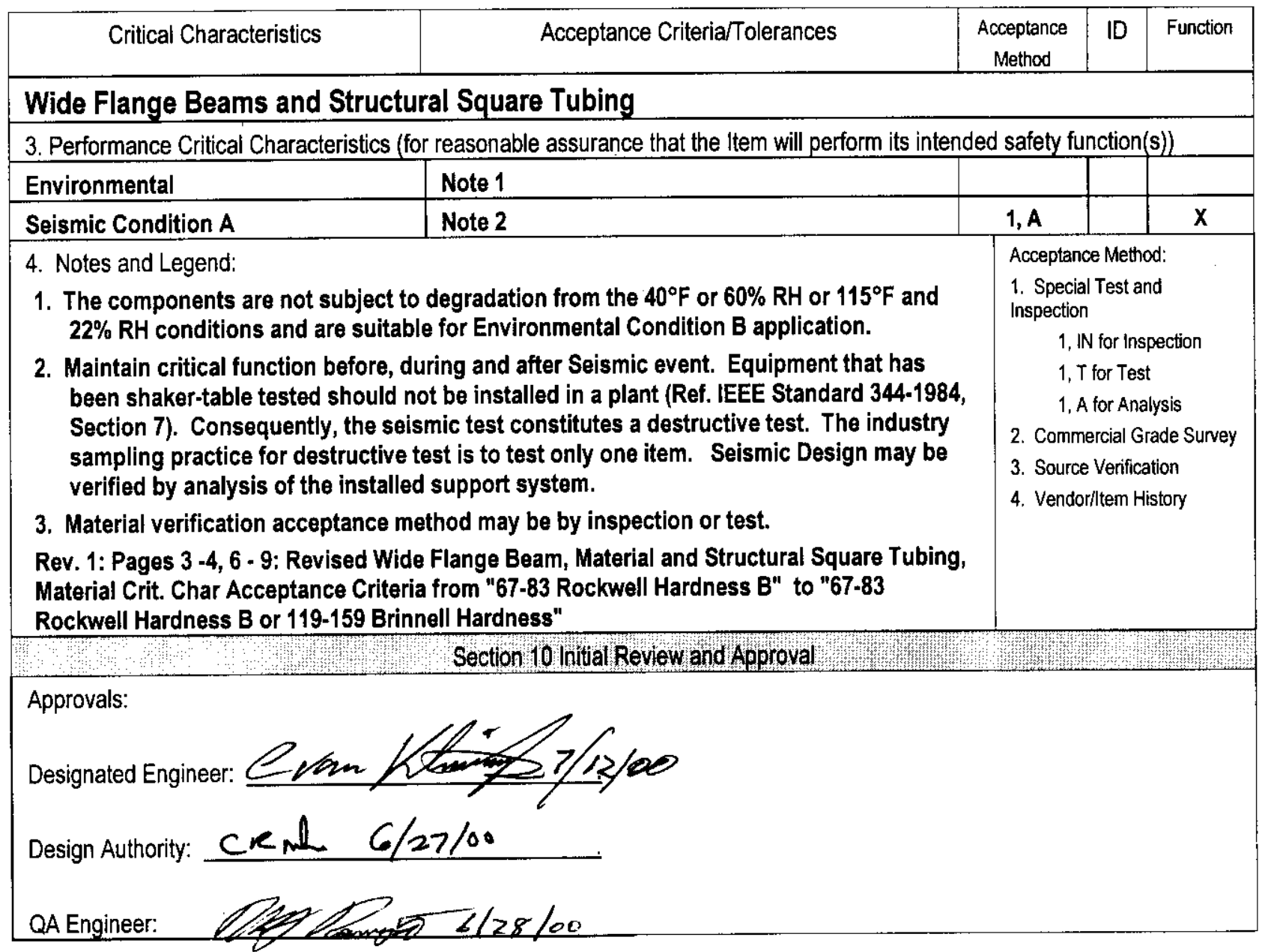

$6 / 27 / 00$ 


\section{WORKSHEET 1}

DETERMINATION OF FAILURE MECHANISMS

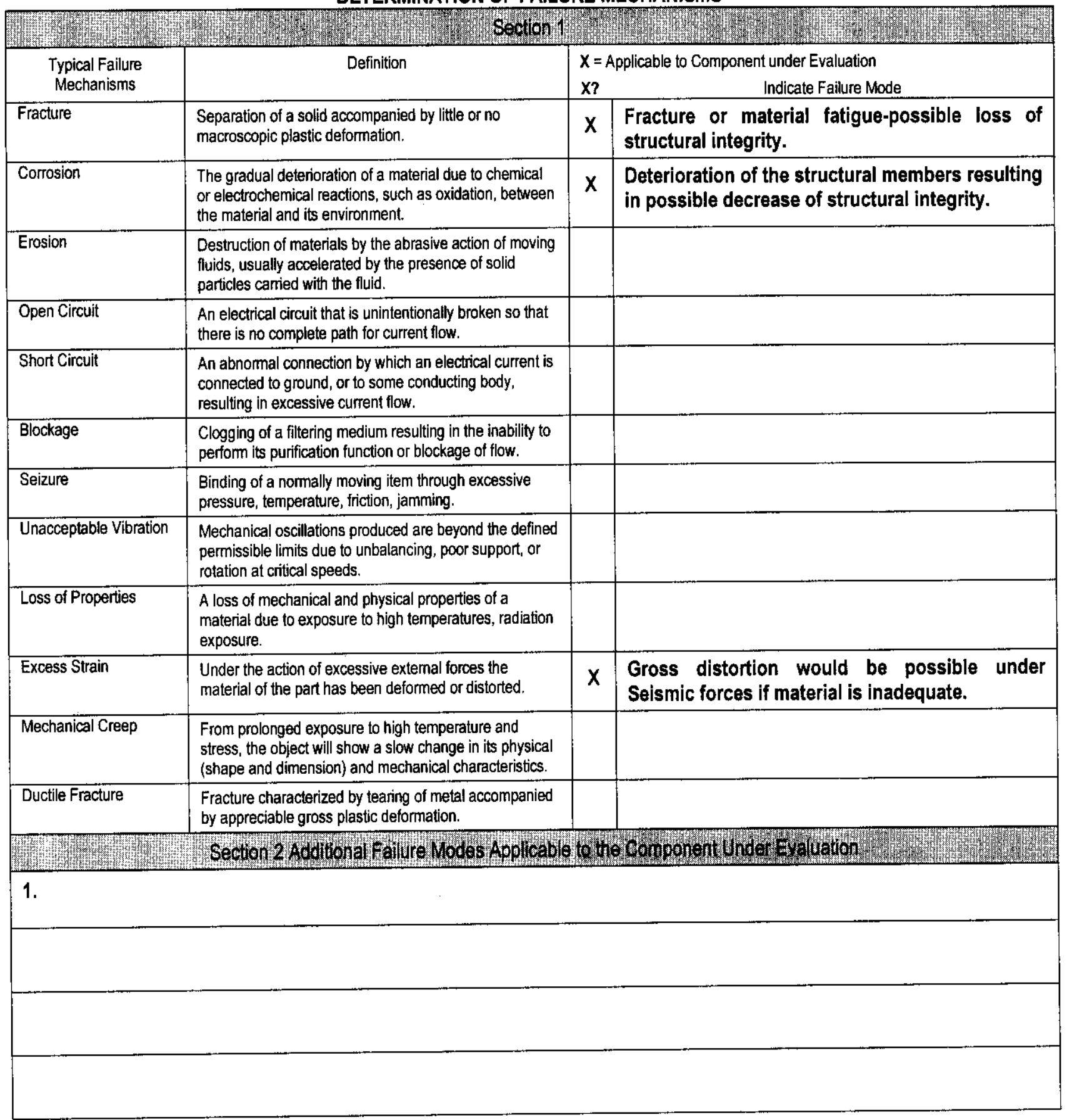


Checklist 1 - Acceptance Method 1 - Special Test/lnspection Verification

\begin{tabular}{|c|c|c|c|}
\hline & & & SETION W \\
\hline \multicolumn{3}{|c|}{$\begin{array}{l}\text { Item Description: Structural steel shapes for process } \\
\text { hood stand. Wide flange beams and structural } \\
\text { square tubing. } \\
\text { System \#: } 30-3\end{array}$} & $\begin{array}{l}\text { Equip \#: NIA } \\
\text { Procurement and/or Part\#: See Characteristics in Section } 9\end{array}$ \\
\hline \multicolumn{3}{|c|}{$\begin{array}{l}\text { Manufacturer (Address/Phone): } \\
\text { ee Section } 9 \text {. }\end{array}$} & $\begin{array}{l}\text { Supplier (Address/Phone): } \\
\text { TBD }\end{array}$ \\
\hline \multicolumn{4}{|c|}{ SECTION 2 CRITICAL CHARACTERISTICS TO BE VERIFIED BY METHOD 1.} \\
\hline \multicolumn{4}{|c|}{$\begin{array}{lll}\text { Insp } & \text { Test } & \begin{array}{c}\text { Post. } \\
\text { Test }\end{array} \\
\end{array}$} \\
\hline$X$ & & \multicolumn{2}{|l|}{ 1. Manufacturer } \\
\hline $\mathbf{X}$ & & \multicolumn{2}{|c|}{ 2. Component Number-Procurement and/or Part Number } \\
\hline$x$ & & \multicolumn{2}{|c|}{ 3. Dimensions (Depth, Width, Length, Thickness) } \\
\hline$x$ & & \multicolumn{2}{|c|}{ 4. Body Material (Verification by inspection of CMTRs) } \\
\hline & $\mathrm{X}$ & \multicolumn{2}{|c|}{ 5. Material - Rockwell Hardness B or Brinnell Hardness } \\
\hline & & \multicolumn{2}{|c|}{ 6. Seismic Condition $C(A=$ Analysis $)$} \\
\hline \multicolumn{4}{|c|}{ 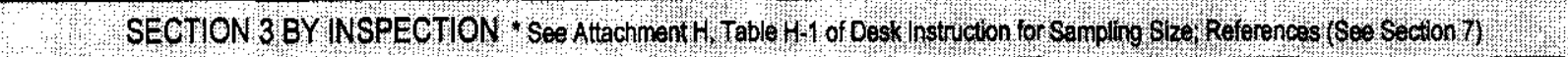 } \\
\hline \multicolumn{4}{|c|}{ Wide Flange Beams } \\
\hline \multicolumn{4}{|c|}{$\begin{array}{l}\text { Characteristic: Manufacturer } \\
\text { Acceptance Criteria: Monarch Machine and Tool } \\
\text { Receipt Inspection Plan / Report \#: }\end{array}$} \\
\hline \multicolumn{4}{|c|}{$\begin{array}{l}\text { Characteristic: Component Number-Procurement andlor Part Number } \\
\text { Acceptance Criteria: Confirm presence of manufacturer's documentation of material compliance with ASTM A 500-93. } \\
\text { Confirm presence of report of the chemical and tensile tests. W10 X } 100 \text { and W8 X } 28 \\
\text { Receipt Inspection Plan / Report \#: }\end{array}$} \\
\hline \multicolumn{4}{|c|}{ 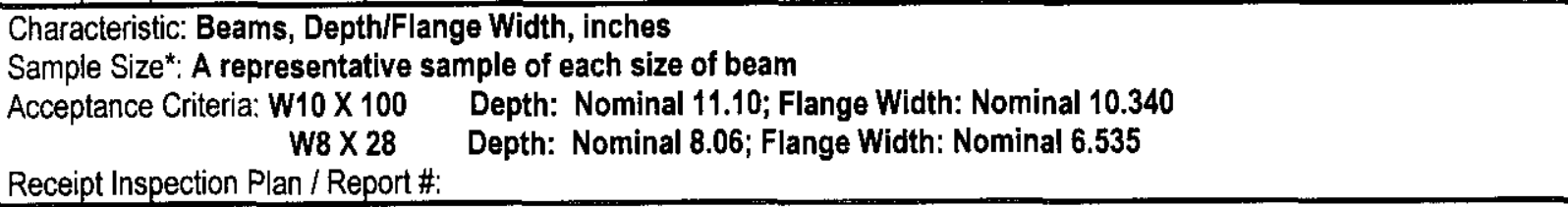 } \\
\hline \multicolumn{4}{|c|}{$\begin{array}{l}\text { Characteristic: Beams, Web Thickness / Flange Thickness } \\
\text { Sample Size*: A representative sample of each size of beam } \\
\text { Acceptance Criteria: W10 X } 100 \quad \text { Web Thickness: Nominal 0.680; Flange Thickness: } 1.120 \\
\text { W8 X } 28 \quad \text { Web Thickness: Nominal } 0.285 \text {; Flange Thickness: } 0.465 \\
\text { Receipt Inspection Plan / Report \#: }\end{array}$} \\
\hline \multicolumn{4}{|c|}{$\begin{array}{l}\text { Characteristic: Material Sample Size } \\
\text { Acceptance Criteria: A-36 Steel - Refer to Manufacturer's documentation of material and report of chemical tests. } \\
\text { Receipt Inspection Plan / Report\#: }\end{array}$} \\
\hline
\end{tabular}




\begin{tabular}{|l|l|} 
Commercial Grade Item Upgrade Dedication Form & SNF-5953, Rev. 1 \\
\hline $\begin{array}{l}\text { ECN No. NIA CGINo. CGI-SNF-D-3-C1-057 } \\
\text { Title: Process Hood Stand Support Steel }\end{array}$ & Page 7 of 10 \\
\hline
\end{tabular}

\begin{tabular}{|c|}
\hline Structural Square Tubing \\
\hline $\begin{array}{l}\text { Characteristic: Manufacturer } \\
\text { Acceptance Criteria: Monarch Machine and Tool } \\
\text { Receipt Inspection Plan / Report \#: }\end{array}$ \\
\hline $\begin{array}{l}\text { Characteristic: Component Number-Procurement and/or Part Number } \\
\text { Acceptance Criteria: Confirm presence of manufacturer's documentation of material compliance with ASTM A 500-93. } \\
\text { Confirm presence of report of the chemical and tensile tests. } 8 \times 8 \times 5 / 8 ; 6 \times 6 \times 1 / 2 ; 3 \times 3 \times 5 / 16 \\
\text { Receipt inspection Plan / Report \#: }\end{array}$ \\
\hline $\begin{array}{l}\text { Characteristic: Outside Dimension / Wall Thickness, inches } \\
\text { Sample Size*: A representative sample of each size of tubing } \\
\text { Acceptance Criteria: } 8 \times 8 \times 5 / 8 \text { Outside Dim: Nominal 8; Wall Thickness: Nominal } 0.625 \\
\qquad \begin{array}{l}6 \times 1 / 2 \text { Outside Dim: Nominal 6; Wall Thickness: Nominal } 0.5000 \\
3 \times 3 \times 5 / 16 \text { Outside Dim: Nominal 3; Wall Thickness: Nominal } 0.3125\end{array} \\
\text { Receipt Inspection Plan / Report\#: }\end{array}$ \\
\hline $\begin{array}{l}\text { Sample Size }: 100 \% \\
\text { Acceptance Criteria: A-36 Steel - Refer to Manufacturer's documentation of material and report of chemical tests. } \\
\text { Receipt Inspection Plan / Report \#: }\end{array}$ \\
\hline 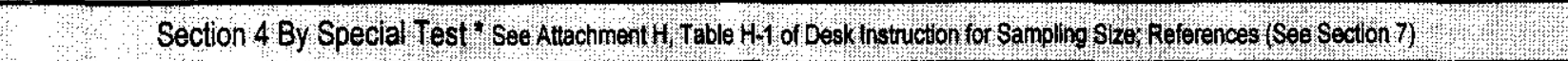 \\
\hline Wide Flange Beams and Structural Square Tubing \\
\hline $\begin{array}{l}\text { Characteristic for Test: Seismic Condition C - All items } \\
\text { Acceptance Criteria: Maintain critical function before, during and after Seismic event. } \\
\text { Sample Size*: Sample size is N/A because seismic verification of the installed support system with structural members } \\
\text { and fasteners, etc., will be done by analysis. Physical testing is not anticipated. } \\
\text { Actual Test Value: } \\
\text { Test Plan and Report \#: }\end{array}$ \\
\hline $\begin{array}{l}\text { Characteristic for Test: Beam Material } \\
\text { Acceptance Criteria: } 67-83 \text { Rockwell Hardness B or 119-159 Brinnell Hardness } \\
\text { Sample Size*: a representative sample of each size of beam. } \\
\text { Actual Test Value: }\end{array}$ \\
\hline $\begin{array}{l}\text { Characteristic for Test: Structural Square Tubing Material } \\
\text { Acceptance Criteria: } 67-83 \text { Rockwell Hardness } \mathrm{B} \text { or } 119-159 \text { Brinnell Hardness } \\
\text { Sample Size*: a representative sample of each size of tubing. } \\
\text { Actual Test Value: }\end{array}$ \\
\hline
\end{tabular}

**If Supplier/Manufacturer or Other, Refer to CGI Checklist-2 for Support Information 


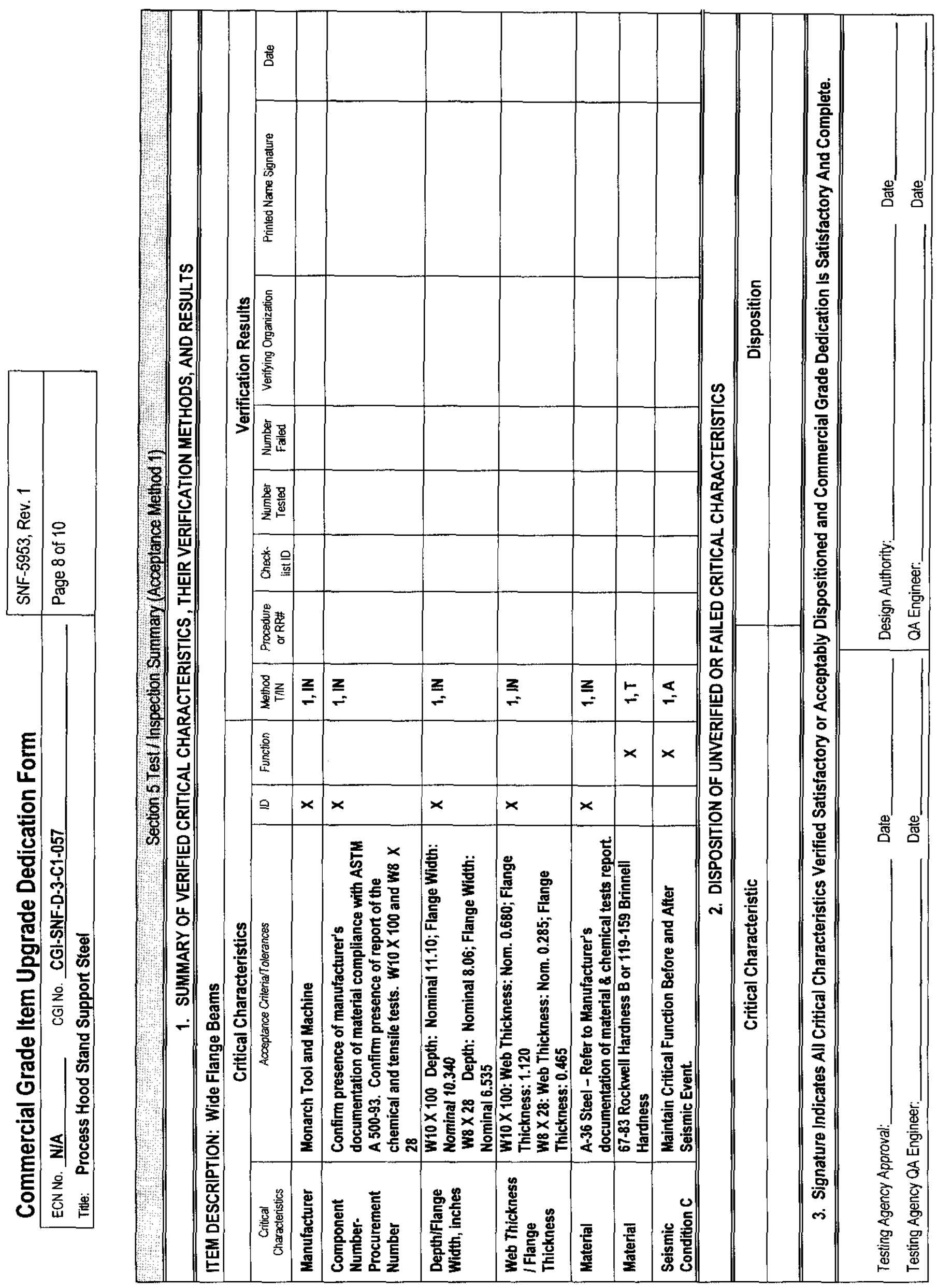




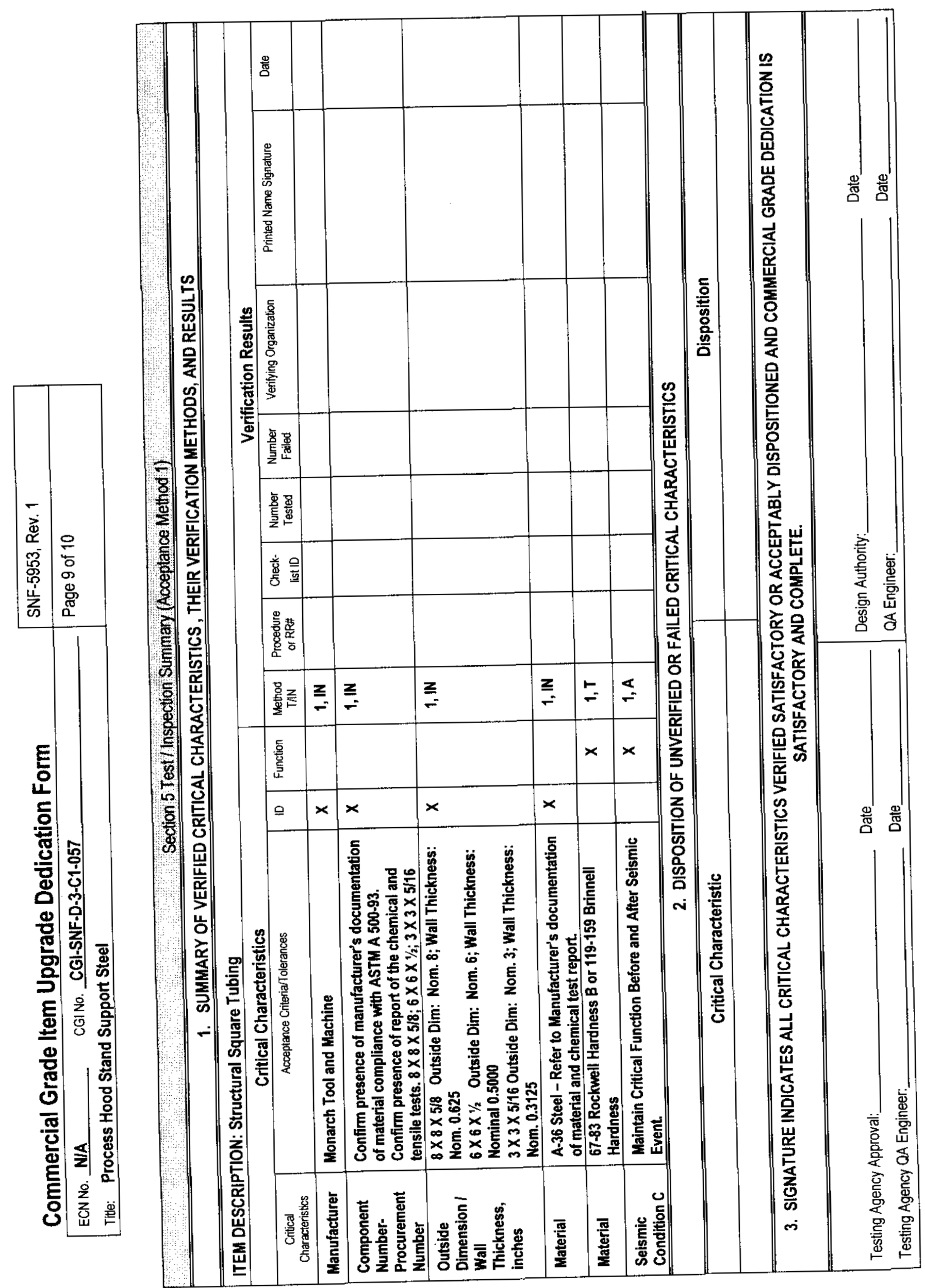


Commercial Grade Item Upgrade Dedication Form

ECN No. N/A

CGI No. CGI-SNF-D-3-C1-057

SNF-5953, Rev. 1

Title: Process Hood Stand Support Steel

Page 10 of 10

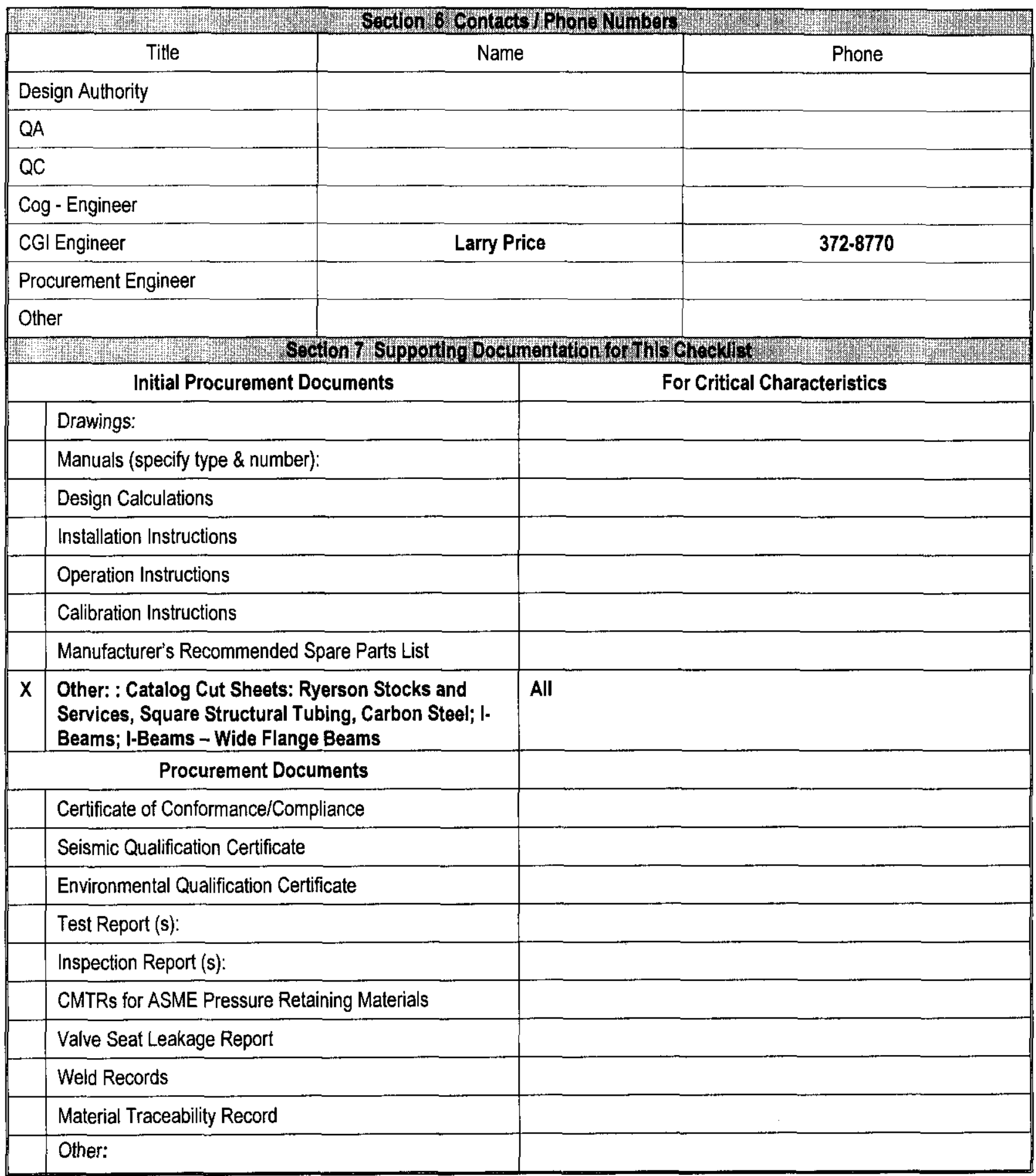




\section{DISTRIBUTION SHEET}

\begin{tabular}{|l|l|}
\hline $\begin{array}{l}\text { To } \\
\text { Distribution }\end{array}$ & $\begin{array}{l}\text { From } \\
\text { SNF-CVD }\end{array}$ \\
\hline
\end{tabular}

Project Title/Work Order

W-44I, CGI Package P4-004 and Cl-057

\section{Whitehurst}

G. Singh

CVD Library

R. Ramsgate

J. Brehm

P. Beaudet

P. Morrell (AVS)

M. Evarts (AI)

L. Price

SNF Startup

SNF Project Files

SNF Satelite Library

C. Van Katwijk

D. Whitworth

T. Nuxall

C. Miska
Page 1 of 1

Date $7 / 13 / 00$

EDT No. NA

ECN No. 661411

\section{Name}

\begin{tabular}{|c|c|c|c|}
\hline MSIN & $\begin{array}{c}\text { Text } \\
\text { With All } \\
\text { Attach. }\end{array}$ & Text Only & $\begin{array}{c}\text { Attach./ } \\
\text { Appendix } \\
\text { Only }\end{array}$ \\
\hline & & & \\
\hline
\end{tabular}

EDT/ECN

Only

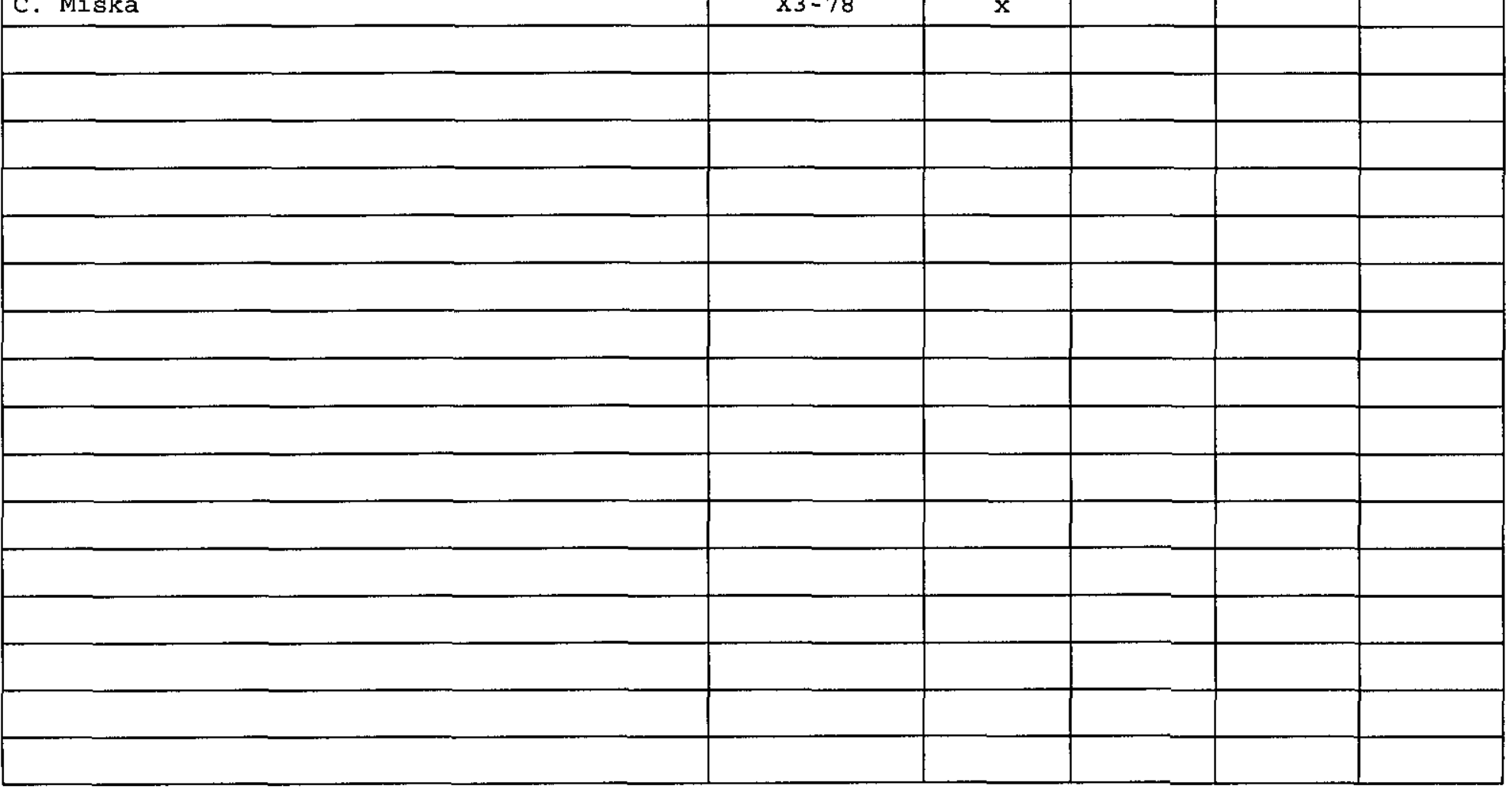

